\title{
Designpreis für Sinterofen
}

Der Sinterofen CEREC SpeedFire von Dentsply Sirona CAD/CAM erhielt die begehrte Design-Auszeichnung. Die Preisverleihung fand kürzlich im Essener Aalto Theater statt. Der seit 5 Monaten erhältliche Induktions-Ofen CEREC SpeedFire ist Teil des CEREC Zirconia Workflows, mit dem Zahnärzte ihren Patienten jetzt den Hochleistungswerkstoff Zirkonoxid chairside anbieten können. Das kompakte und anwenderfreundliche $\mathrm{Ge}$ rät vereint dabei die Prozesse Sintern und Finalisieren (Glasieren). Beide Prozesse nehmen nur wenige Minuten in Anspruch: Eine Krone ist beispielsweise nach 10-15 min gesintert und nach 9 min glasiert. Aufgrund seiner Verbindung mit

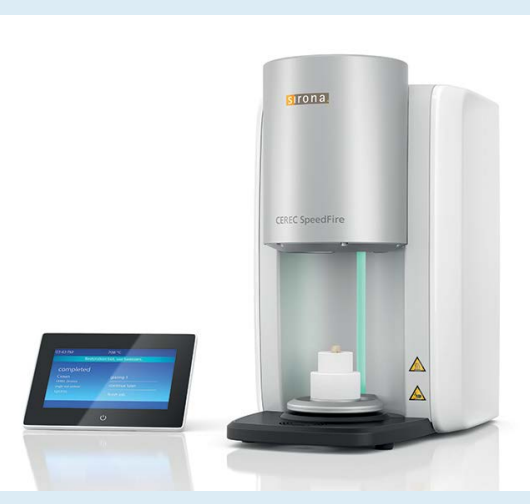

dem System verfügt der Ofen über alle notwendigen Informationen zu Material, Farbe, Typ und Umfang der Restauration aus der CEREC Software von Dentsply Sirona CAD/CAM. Spezielle Schulungen oder lange Übungsprozesse sind nicht erforderlich. Mit dem weltweit anerkanntem Red Dot Award werden seit 1954 Produkte, Designkonzepte und Kommunikationsdesigns prämiert.

Nach einer Pressemitteilung der

Dentsply Sirona CAD/CAM, A - Wals bei

Salzburg 